\title{
AMON: Multimessenger Alerts from High-Energy Gamma Rays and Neutrinos
}

\author{
Hugo Alberto Ayala Solares* \\ The Pennsylvania State University \\ E-mail: hgayalaepsu.edu
}

\section{for the AMON Project}

\begin{abstract}
The Astrophysical Multimessenger Observatory Network (AMON) has been working to link the world's high-energy and multimessenger observatories together into a single network in order to evoke discovery of multimessenger sources, exploit these sources for purposes of astrophysics, fundamental physics, and cosmology, and explore project datasets for evidence of multimessenger source populations. AMON has been working to commission multiple multimessenger alert streams, including gravitational wave + gamma-ray $(\mathrm{GW}+\gamma)$ and high energy neutrino + gammaray $(v+\gamma)$ coincidence alerts. One such $v+\gamma$ alert stream, now in an advanced stage of development, will search in near real-time for statistically-rare coincidences between $\sim \mathrm{TeV}$ gamma-rays observed by the High-Water Altitude Cherenkov Observatory (HAWC) and $\gtrsim \mathrm{TeV}$ neutrinos detected by the IceCube Neutrino Observatory. We describe the statistical design, calibration, and validation of these HAWC and IceCube $v+\gamma$ alerts, which will be commissioned soon and made available to AMON follow-up partners under terms of the AMON MoU. With a median delay to alert distribution of six hours and angular uncertainties of $\lessgtr^{\circ}$, the alerts should be well-suited for deep electromagnetic follow-up observations.
\end{abstract}

The New Era of Multi-Messenger Astrophysics - Asterics2019

25 - 29 March, 2019

Groningen, The Netherlands

${ }^{*}$ Speaker. 


\section{Introduction}

Two events during 2017 marked the dawn of a new era of multimessenger astrophysics. First, the detection of gravitational waves from the coalescence of a binary neutron star system by the LIGO+Virgo detectors, accompanied by a short gamma-ray burst detected by the Fermi-GBM telescope, and followed up by a wide array of observatories and detectors around the globe [1]. Second, the observation of a high-energy neutrino by the IceCube detector, which was then shown to be correlated spatially and temporally with a gamma-ray flaring episode of the blazar TXS $+0506+056$, as observed by Fermi-LAT and the MAGIC telescope [2].

These events have motivated the scientific community to keep pursuing multimessenger observations, specifically in realtime. The combination of different datasets is a powerful way to use the information from different messengers, which ultimately shades a light on the different models that scientists have to explain the astrophysical phenomena. However, the field is new and several challenges, such as the coordination, communication and accords have to be made in order for the field to grow.

The Astrophysical Multimessenger Observatory Network (AMON) has been created with the purpose of facilitating the interaction of the different observatories, creating a framework capable of doing analysis with different datasets, and alerting the scientific community of any interesting events. In this proceeding, we present a brief overview of what AMON is and also one of the analysis that is being developed within AMON for looking for coincidences of high-energy gamma rays and neutrinos.

\section{The Astrophysics Multimessenger Observatory Network (AMON)}

AMON is a virtual observatory network designed to receive events from different detectors and perform coincidence analysis in realtime in order to produce alerts that can be followed-up by AMON members and the community in general.

AMON's network is formed by triggering observatories - which have large field of view and have monitoring capabilities - and follow-up observatories, generally telescopes designed to point at specific points in the sky which can carry out follow-up observations of alerts from other detectors. A list of the current members of AMON can be found in Table 2 of [4].

Potential signal events which are difficult to be distinguished from the background of individual detectors, can be recovered if these events also appear in the datasets from other observatories and careful statistical analysis are performed. We refer to this events as sub-threshold events.

AMON's main goals are to perform coincidence searches of sub-threshold events from different observatories in realtime; receive events and broadcast them through the Gamma-Ray Coordinates Network; and store events to perform archival searches for significant coincidences.

AMON is currently hosted by the Pennsylvania State University. The two main production servers are located at the Institute for CyberScience (ICS) of the university. These two servers have an up-time of $>99.99 \%$, meaning less than one hour of downtime per year. The servers are in charge of the real-time system, storage of the events and computation of the coincidence algorithms. A virtual machine, hosted by the Advanced CyberInfrastructure (ACI) of the ICS helps the AMON group to develop and test the software and system deployed in the production 
machines. The software used by AMON is written in Python 2.7 and its repository resides in the GitHub webpage https://github.com/AMONCode/Analysis.

AMON's plan is to generate multiple multimessenger channels, producing coincidence alerts for follow-up observations. The majority of the AMON analyses will be based on a likelihood method (with differences depending on the information the detectors and observatories agree to release). An example of one of these analyses is shown in [5] and below.

\section{HAWC and IceCube $v+\gamma$ alerts}

The IceCube observatory is a detector of high-energy neutrinos located at the south pole. The detector construction started in 2005 and finished in 2010. During 2017, evidence was found that the IceCube event 170922A was coincident with gamma-ray emission from the blazar TXS $0506+056$.

The HAWC observatory is a high-energy gamma-ray detector, located in central Mexico. HAWC has a large field of view, covering two thirds of the sky daily and a high-duty cycle. HAWC has found interesting objects, such as the TeV jets from SS 433 and a new class of halos, named TeV-Halos, such as Geminga.

We perform a coincidence analysis which will use data from both observatories. The analysis is currently under review by both collaborations and it is expected to be issuing near real-time alerts on the second part of 2019.

\subsection{Datasets}

HAWC data consists of what is termed a "hotspot." This is a significant excess above the background averaged over 1 transit of the event above the detector. The main event parameters AMON receives are: the position and its uncertainty, significance value $(>2.75 \sigma)$ and the start and stop times of the transit. The cut in the significance was defined by the HAWC collaboration to send sub-threshold events. Current rate of these events received by AMON is of $\sim 800$ per day.

The IceCube data consists of single through-going track events, mostly produced by CC muon neutrinos. The parameters of these events that are sent to AMON are: position and its uncertainty, time of the event, false positive rate density and signal acceptance. Current rate of these events received by $\mathrm{AMON}$ is of $\sim 650$ per day.

\subsection{Statistical Design}

For this analysis, the coincidence is defined by two criteria. The first is a temporal one, where we look for neutrinos inside the transit time of the HAWC "hotspot". The second criteria is to select neutrinos that are close to the HAWC "hotspot" by $3.5^{\circ}$. After the events have passed the selection criteria, we perform the calculation of a ranking statistic in order to select the best coincident events. This ranking statistic is based on Fisher's method, were we combine all the information that we have from the events. This is performed as

$$
\chi_{6+2 n_{v}}^{2}=-2 \ln \left[p_{\lambda} p_{\text {HAWC }} p_{\text {cluster }} \prod_{i}^{v} p_{I C, i}\right]
$$


where $p_{\lambda}$ quantifies the overlap of the uncertainties of the events; $p_{\text {HAWC }}$ is the p-value of the HAWC event been from background; $p_{\text {cluster }}$ is the probability of seeing more than one neutrino in the HAWC transit period; and $p_{I C, i}$ is the probability of the IceCube event been from background. The only p-value that we have to calculate is the $p_{\lambda}$, which comes from maximizing a likelihood calculation that measures how much the position of the HAWC event and the IceCube events overlap with each other. This is calculated as

$$
\lambda(\vec{x})=\sum_{i=1}^{N} \ln \left(S_{i}(\vec{x})\right)-\ln \left(B_{i}\right),
$$

where $S$ corresponds to the uncertainties of the events, assuming Gaussian distributions on the sphere, and $B$ is the spatial background distribution from each detector at the position of the events. This likelihood is maximized by finding the best position of the coincidence $\vec{x}$. A bigger $\lambda$ value means the uncertainties of the events overlap more, which in turn translates into a smaller $p_{\lambda}$.

Due to the fact that we can have more than one neutrino in the time window, this affects the degrees of freedom of equation 3.1. Considering this, we transform the $\chi^{2}$ to a $p$-value, with the corresponding number of degrees of freedom, and then perform the minus logarithm of this quantity. This is represented as

$$
\chi^{2^{\prime}}=-\log p\left(>\chi_{6+2 n_{v}}^{2}\right)
$$

which is the value that we used to rank the coincidences.

\subsection{Calibration}

We applied this algorithm to a 2-year dataset from both detectors, and scrambled the datasets several times for calibration of the analysis. Scrambling is performed by randomly permuting the R.A. information of the events. Parameters that are declination dependent were kept together with their respective declination (e.g. false positive rate density in IceCube, and transit time in HAWC). The distribution of the coincidences of equation 3.3 is shown in Figure 1a. The false alarm rate as a function of $\chi^{2^{\prime}}$ is shown in Figure $1 b$.

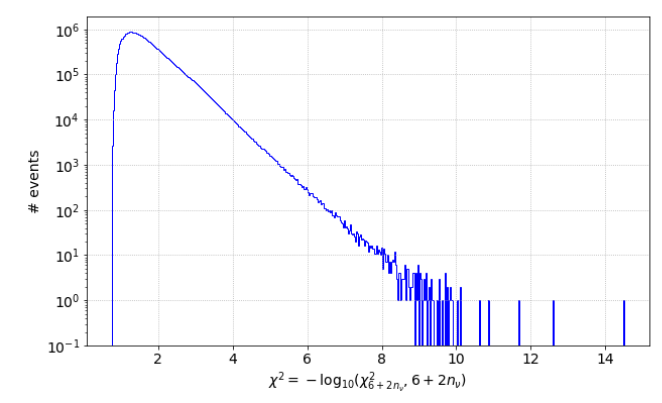

(a) Distribution of random coincidences from the scrambled datasets. This is equivalent to 725 years of data.

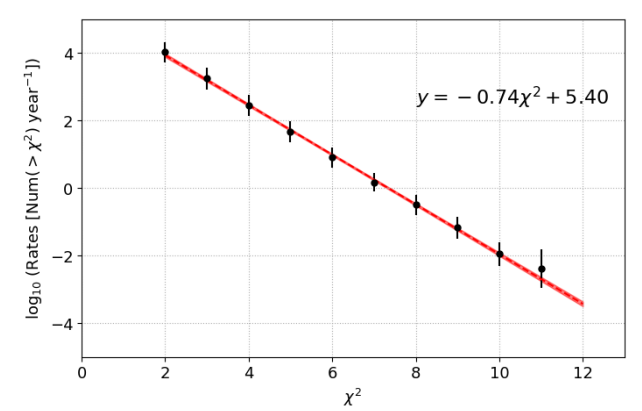

(b) False alarm rate as a function of the ranking statistic. This is the main function that will be used to select alerts that will be sent to GCN.

Figure 1: Scrambled analysis distributions

These figures will be our reference for when we start issuing coincidence alerts from this analysis. In general, we plan to send alerts with a false alarm rate of $<1$ per year, although we 
do not rule out issuing alerts with higher false alarm rates, depending on the interest of AMON follow-up partners.

Finally, we plan to validate our analysis by carrying out an archival search for coincidences using the unscrambled HAWC and IceCube datasets.

\section{Towards the future}

The field of multimessenger astrophysics will continue to grow thanks to the new technologies and the different instruments that are under development for the detection of the different messengers of the four forces of nature. The cooperation between observatories and detectors will be crucial for this task. AMON is tailored for this important task, enabling real-time and archival searches for multimessenger sources. The gamma-ray and neutrino multimessenger channel will be an interesting channel to look at since it will help us look for sources that hint to hadronic processes. The HAWC plus IceCube coincidence analysis is aimed for this task. AMON's plan is to send any coincidence alert from this stream that has a false alarm rate of $<1$ per year to the community through GCN.

\section{Acknowledgments}

This research or portions of this research were conducted with Advanced CyberInfrastructure computational resources provided by the Institute for CyberScience (https://ics.psu.edu) at the Pennsylvania State University .

This material is based upon work supported by the National Science Foundation under Grants PHY1708146 and PHY-1806854 and by the Institute for Gravitation and the Cosmos of the Pennsylvania State University. Any opinions, findings, and conclusions or recommendations expressed in this material are those of the author(s) and do not necessarily reflect the views of the National Science Foundation.

\section{References}

[1] L. S. Collaboration, V. Collaboration, et al., Multi-messenger Observations of a Binary Neutron Star Merger, ApJL, 848, 59, 2017.

[2] IceCube Collaboration, Fermi-LAT, MAGIC, et al., Multimessenger observations of a flaring blazar coincident with high-energy neutrino IceCube-170922A, Science, 361, 8, 2018.

[3] M. Smith, et al., The Astrophysical Multimessenger Observatory Network (AMON), Astro. Part. 45, 56, 2013.

[4] H. A. Ayala Solares, et al., The Astrophysical Multimessenger Observatory Network (AMON), arXiv:180, 2018.

[5] C. F. Turley, D. B. Fox, K. Murase, et al., Search for Blazar Flux-correlated TeV Neutrinos in IceCube 40-string Data, ApJ, 833, 117, 2016.

[6] AMON website http://amon.psu.edu/ 